\section{Overcoming C. differences}

\section{By Lev Osherovich, Senior Writer}

It has been known for some time that the pathogenesis of Clostridium difficile depends on a pair of toxic proteins secreted by the bacterium. But it has been less clear which of the two toxins-toxin A or toxin B-represents a better therapeutic target. Now, a report by British researchers makes a case that the toxins should be jointly targeted. ${ }^{1}$

The findings, which were published in Nature, support the dual toxin-targeting strategies behind $\mathrm{mAb}$-based therapies being pursued by Merck \& Co. Inc., which has MK-3415A, a coformulation of mAbs against toxins A and B, and by Progenics Pharmaceuticals Inc., which has preclinical mAbs targeting both toxins.

Toxins A and B are large proteins that penetrate into epithelial cells and disrupt intracellular signaling and cellular structure, leading to fluid accumulation, inflammation, diarrhea and sepsis. The two proteins account for $C$. difficile's virulence and thus are attractive targets for treating the acute diarrhea that often occurs when the bacterium colonizes intestines stripped of their natural microbes by antibiotics.

"It was originally thought that toxin A was the most important [virulence factor], but as time went on people focused on toxin B" because of the discovery of virulent strains lacking toxin A, said Nigel Minton, professor of applied molecular microbiology at The University of Nottingham and senior author of the Nature paper. "We've re-established the importance of toxin A in disease."

\section{Strained relationship}

Minton's team began by creating $C$. difficile strains with targeted disruptions of the genes encoding toxin $\mathrm{A}$, toxin $\mathrm{B}$ or both toxins and testing the strains in vitro.

Bacterial culture media exposed to wild-type $C$. difficile killed human and African green monkey cell lines, whereas media exposed to strains lacking both toxins were harmless. The fluids from toxin A-deficient but toxin B-containing bacterial cultures and from toxin B-deficient but toxin A-containing cultures both killed the primate cell lines, indicating that each toxin was potent.

The team then tested mutant $C$. difficile strains in a hamster model of intestinal infection. Hamsters purged of their natural gut biota by a course of antibiotics proved susceptible to infection by wild-type C. difficile and died about a day after inoculation. Toxin A and toxin B single knockout strains killed hamsters after 1.3 and 4 days, respectively, whereas a double toxin A and B knockout strain was avirulent.

The findings run counter to a 2009 Nature report by Australian researchers. ${ }^{2}$ In that study, a team led by Dena Lyras, senior lecturer in the Department of Microbiology at Monash University, isolated toxin A- and toxin B-deficient strains. The group found that, unlike toxin A-deficient strains, bacteria lacking toxin B did not readily kill hamsters. Thus, Lyras' team concluded that toxin B was more important than toxin A in infection in vivo.

"In Minton's study, they found that if you take away toxin B you don't reduce virulence by much, which is different than what we found," said Lyras.

\section{Toxin targets}

Sorting out why the two teams got divergent results may help resolve whether it's necessary to target both toxins, as Minton's study suggests, or whether hitting toxin B will be enough, as implied by Lyras' work.

"If you're designing an antibody as a therapeutic for $C$. difficile infection, you'd want to pick toxin B" as a target, Lyras told SciBX. She cited clinical reports of toxin A-deficient strains that were nonetheless highly pathogenic, arguing that toxin B is sufficient for toxicity.

Lyras noted that there are many variants of toxin $B$, with different effects in various tissues, so having a diverse array of mAbs against toxin $B$ could be broadly useful.

Donna Ambrosino, executive director of Massachusetts Biologic Laboratories, a not-for-profit vaccine and antibody manufacturer operated by the University of Massachusetts Medical School, came down in favor of Minton's interpretation. Ambrosino headed a Phase II trial of MK-3415A, which was developed by Massachusetts Biologic Laboratories and Bristol-Myers Squibb Co.'s Medarex Inc. unit. ${ }^{3}$

In that study, MK-3415A met the primary endpoint of preventing recurrence of $C$. difficile-associated diarrhea (CDAD).

MK-3415A is licensed to Merck.

"We're biased by our clinical data in that we strongly feel that antibodies to both toxin A and toxin B are necessary to prevent disease in patients," Ambrosino said.

She added that her own team's work in hamsters and in clinical testing of components of MK-3415A agree with Minton's finding that either of the two toxins can cause disease.

"We went on to study what happens when you give one antibodyagainst toxin A alone-to patients," said Ambrosino. The treatment, she said, "showed no benefit," suggesting that toxin B also needs to be targeted.

Ambrosino said the reciprocal experiment of targeting toxin B alone in humans has not yet been attempted.

"Perhaps [Lyras'] strains are a bit attenuated and are making only one toxin," said Ambrosino. "Neither paper is exactly wrong, but it may be a matter of how the models, strains and dosing of the toxin are different."

According to Lyras, one possible explanation for the disagreement between the studies may be a minor difference in the survival assays used by the two teams. Minton suggested that Lyras' findings potentially could be explained by secondary mutations in the strains used by the Australian team. 


\section{ANALYSIS}

\section{TARGETS \& MECHANISMS}

\section{Face the strain}

Regardless of the disagreement, the most immediate value of both studies may be in the knockout strains themselves. In vitro and animal testing against strains lacking either toxin could help companies optimize $\mathrm{mAb}$ combinations.

"Both of these papers are very important," said William Olson, SVP of R\&D at Progenics. "The tools that they've generated are extremely useful for the field. We've never had these isogenic strains before."

Earlier this month, Progenics reported in vitro and hamster data for its mAbs against toxin A and toxin B at the International Conference on Antimicrobial Agents and Chemotherapy. According to Olson, the Progenics mAbs bind to both toxins through a different mechanism than the mAbs in Merck's MK-3415A and thus display higher affinity in vitro than Merck's mAbs.

Olson said Progenics' high-affinity mAbs could be used to prevent the initial appearance $C$. difficile-associated disease rather than prevent recurrence.

Neither Minton nor Lyras patented their findings. Lyras said she is collaborating with an undisclosed company to develop therapeutics and diagnostics based on her discoveries.

Osherovich, L. SciBX 3(37); doi:10.1038/scibx.2010.1112

Published online Sept. 23, 2010

REFERENCES

1. Kuehne, S.A. et al. Nature; published online Sept. 15, 2010; doi:10.1038/nature09397

Contact: Nigel P. Minton, The University of Nottingham, Nottingham, U.K. e-mail: nigel.minton@nottingham.ac.uk

Contact: Stephen Cartman, same affiliation as above e-mail: stephen.cartman@nottingham.ac.uk

2. Lyras, D. et al. Nature 458, 1176-1179 (2009)

3. Lowy I. et al. N. Engl. J. Med. 362, 197-205 (2010)

COMPANIES AND INSTITUTIONS MENTIONED

Bristol-Myers Squibb Co. (NYSE:BMY), New York, N.Y. Massachusetts Biologic Laboratories, Worcester, Mass. Medarex Inc. (NASDAQ:MEDX), Princeton, N.J.

Merck \& Co. Inc. (NYSE:MRK), Whitehouse Station, N.J.

Monash University, Melbourne, Australia

Progenics Pharmaceuticals Inc. (NASDAQ:PGNX), Tarrytown, N.Y. University of Massachusetts Medical School, Worcester, Mass. The University of Nottingham, Nottingham, U.K. 